GENETICS

\title{
Bridging the divide
}

Children with Down's syndrome (DS) have an increased risk of developing leukaemias particularly acute megakaryoblastic leukaemia (AMKL) - but the link between DS and cancer is not well understood. In the September issue of Nature Genetics, Wechsler et al. report that people with DS who develop AMKL all carry a somatic mutation in a gene encoding a transcription factor that is required for normal haematopoiesis.

Wechsler et al. started out studying a gene called GATA1, which encodes a zinc-finger transcription factor that is known to be required for growth and maturation of erythroid cells and megakaryocytes. Inherited mutations in the zincfinger domain of GATA1 had been previously reported to cause congenital dyserythropoietic anaemia and thrombocytopaenia, and megakaryocytes that lack GATA1 had been observed to proliferate excessively without

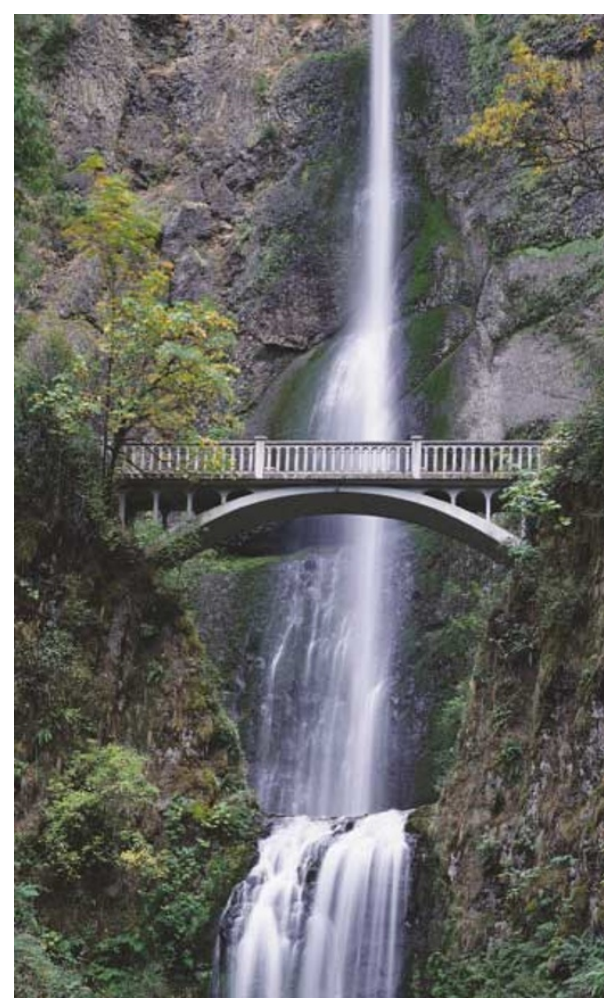

differentiating into platelets. So the authors looked to see if mutations in this gene could also be associated with myeloid leukaemias.

In analysing blast cells from leukaemia patients, the authors detected mutations that introduced a premature stop codon in GATA1 in each of six individuals with DSAMKL. By contrast, they did not detect mutations in GATA1 in DNA from healthy individuals, people with other myeloid-leukaemia subtypes that did not have DS, or people with DS that had other types of leukaemias.

Megakaryoblasts from people with DS-AMKL did not express the full-length GATA1, although they do produce a shorter, alternative translation product from a downstream initiation site.

So why does this particular mutation arise in people with DS? DS is associated with trisomy 21 (three copies of chromosome 21), which leads to the acquisition of somatic-cell mutations on other chromosomes. The authors propose that overexpression of a gene or genes on chromosome 21 might stimulate abnormal proliferation of haematopoietic stem cells. The expanded pool of dividing cells would then be able to acquire mutations in genes on other chromosomes, such as GATA1 (which is on the $\mathrm{X}$-chromosome), and cancers such as AMKL could develop. Analysis of mice with partial trisomy 21 that have been crossed to mice that carry Gata1 mutations will provide additional information about the mechanisms of leukaemogenesis in DS.

Kristine Novak

(2) References and links ORIGINAL RESEARCH PAPER Wechsler, J. et al. Acquired mutations in GATA1 in the megakaryoblastic leukemia of Down Syndrome. megakaryoblastic leukemia of Down Syndrome.
Nature Genet. 12 Aug 2002 (doi:10.1038/ng955). FURTHER READING Look, A. T. A leukemogenic twist for GATA1. Nature Genet. 12 Aug 2002 (doi:10.1038/ng960).

WEB SITE

John Crispino's lab: http://ben-may.bsd. uchicago.edu/CCB/faculty/crispino.html

\section{TRIAL WATCH}

\section{Particle power}

An $\alpha$-particle-emitting isotope coupled to the humanized antiCD33 antibody HuM195 has shown promise in a Phase I cancer trial. This antibody, which specifically targets myeloid leukaemia cells, was previously coupled to the $\beta$-emitters ${ }^{131}$ iodine and ${ }^{90} \mathrm{yttrium}$, and shown to eliminate leukaemic cells in patients. However, it also killed normal bystander cells and induced prolonged myelosuppression. To enhance the potency of the antibody yet avoid the nonspecific cytotoxicity of $\beta$-emitting constructs, Scheinberg and colleagues coupled HuM195 to the $\alpha$-emitting isotope ${ }^{213}$ bismuth. This $\alpha$-particle-emitting isotope has a smaller range than $\beta$-emitters, but still induces DNA damage and apoptosis of nearby cells. Eighteen patients with relapsed and refractory acute myeloid leukaemia or chronic myelomonocytic leukaemia were given ${ }^{213}$ bismuth-HuM195. Although all evaluable patients developed myelosuppression, no significant extramedullary toxicity was seen. Nearly all the

${ }^{213}$ bismuth-HuM195 rapidly localized to and was retained in leukaemic areas. Absorbed dose ratios between these sites and the whole body were 1,000 -fold greater than those seen in patients treated with the $\beta$-emitting constructs. In total, $93 \%$ of evaluable patients had reductions in circulating blasts, and $78 \%$ of patients had reductions in the percentage of bone-marrow blasts. This is the first targeted $\alpha$-particle immunotherapy in humans.

ORIGINAL RESEARCH PAPER Jurcic, J. G. et al. Targeted $\alpha$-particle immunotherapy for myeloid leukemia. Blood 100, 1233-1239 (2002)

\section{Efficacy of imatinib}

Two clinical studies published in the 15 August issue of the New England Journal of Medicine show that durable responses can be achieved in patients who have cancers other than chronic myelogenous leukaemia when treated with imatinib (Glivec). In an open-label, randomized, multicentre Phase II trial, 147 patients with advanced gastrointestinal stromal tumours (GISTs) were treated with imatinib daily. Demitri and co-workers report partial responses in $54 \%$ of patients and a further $28 \%$ of patients with stable disease, although 20 patients did show early resistance to imatinib. The objective responses were durable at a median followup of 24 weeks. The therapy was well-tolerated. Constitutive activation of the KIT receptor tyrosine kinase is crucial in the formation of GISTs and this study shows that imatinib, a selective inhibitor of KIT, is a promising treatment for this disease.

In the second paper, Apperley and colleagues report responses with imatinib in four patients with chronic myeloproliferative disease, which is characterized by the constitutive transcriptional activation of the gene for platelet-derived growth factor receptor- $\beta$ (PDGFR- $\beta$ ). This receptor tyrosine kinase is also inhibited by imatinib. In all four patients, a normal blood count was achieved soon after beginning treatment. The $\mathrm{t}(5 ; 12)$ translocation that caused the constitutive activation of PDGFR- $\beta$ was undetectable within 3 months in all four patients, and transcript levels of the gene also decreased. These responses were durable at 9-12 months of follow-up, indicating a possible role for imatinib in this disease.

ORIGINAL RESEARCH PAPERS Demetri, G. D. et al. Efficacy and safety of imatinib mesylate in advanced gastrointestinal stromal tumours. N. Engl. J. Med. 347, 472-480 (2002) | Apperley, J. F. et al. Response to imatinib mesylate in patients with chronic myeloproliferative diseases with rearrangements of the platelet-derived growth factor receptor- $\beta$. N. Engl. J. Med. 347, 481-487 (2002) 